\title{
Computing the invariant ring of a finite group
}

\author{
THOMAS HAWES
}

\begin{abstract}
We give an overview of a new package for Macaulay2 called InvariantRing, which contains tools for describing the invariant ring of finite group actions on polynomial rings in characteristic zero. We outline methods for computing primary and secondary invariants and compare the two algorithms that are implemented for computing primary invariants.
\end{abstract}

INTRODUCTION. Let $k$ be a field of characteristic zero and $\operatorname{GL}(n, k)$ the group of invertible $n \times n$ matrices. Let $S:=k\left[x_{1}, \ldots, x_{n}\right]$ be the ring of polynomial functions on $k^{n}$, where elements of $k^{n}$ are considered as column vectors. We write $\mathbf{x}=\left(x_{1}, \ldots, x_{n}\right)^{\mathrm{t}}$ for the column vector of the variables of $S$ and let elements of $\operatorname{GL}(n, k)$ act on $\mathbf{x}$ by matrix multiplication. For a finite subgroup $G \leq \operatorname{GL}(n, k)$, consider the left action on $S$ defined by $(A \cdot f)(\mathbf{x}):=f\left(A^{-1} \mathbf{x}\right)$, for any $A \in G$ and $f \in S$. The invariant ring of the group $G$ is the $\operatorname{ring} S^{G}:=\{f \in S \mid A \cdot f=f$ for all $A \in G\}$ and its elements are called invariants. Due to the fact that $G$ acts on $S$ by linear transformations, a polynomial is invariant if, and only if, each of its homogeneous pieces is invariant; thus $S^{G}$ is a graded subring of $S$. The following structure theorem, which can be found in $[\mathrm{N}, \mathrm{DK}]$, provides the main motivation for the package InvariantRing.

Theorem 1. For any finite group $G \leq \mathrm{GL}(n, k)$, there exist homogeneous, algebraically independent invariants $f_{1}, \ldots, f_{n}$ and homogeneous invariants $g_{1}, \ldots, g_{r}$ such that

$$
S^{G}=\bigoplus_{j=1}^{r} k\left[f_{1}, \ldots, f_{n}\right] g_{j} .
$$

The $f_{1}, \ldots, f_{n}$ are called primary invariants and the $g_{1}, \ldots, g_{r}$ secondary invariants. The purpose of the package InvariantRing for Macaulay2 [M2] is to compute primary and secondary invariants for the invariant ring of a finite group over number fields.

Collections of primary and secondary invariants for an invariant ring are not uniquely determined, but once a system of primary invariants has been chosen, the degrees of any corresponding system of secondary invariants are determined by the following theorem (see [DK]):

Theorem 2. Let $f_{1}, \ldots, f_{n}$ be primary invariants for $S^{G}$ with degrees $d_{1}, \ldots, d_{n}$, and let $H\left(S^{G}, t\right) \in \mathbb{Z}[t]$ be the Hilbert series of $S^{G}$. Suppose there are r corresponding secondary invariants.

(a) $H\left(S^{G}, t\right) \cdot \prod_{i=1}^{n}\left(1-t^{d_{i}}\right)=t^{e_{1}}+\cdots+t^{e_{r}}$, where $e_{1}, \ldots, e_{r}$ are the degrees of any corresponding secondary invariants. In particular, this polynomial has integer coefficients.

(b) The number of secondary invariants is $r=\frac{d_{1} \cdots d_{n}}{|G|}$. In particular, $|G|$ divides $d_{1} \cdots d_{n}$. 
It is easy to compute the Hilbert series of $S^{G}$ by using Molien's theorem, which states that $H\left(S^{G}, t\right)=\frac{1}{|G|} \sum_{A \in G} \operatorname{det}\left(I_{n}-t A\right)^{-1}$, where $I_{n}$ is the $n \times n$ identity matrix [N]. Molien's theorem, along with Theorem 2, gives the number of secondary invariants and their degrees.

The package InvariantRing provides tools to study and compute the invariant ring of a finite group action on a polynomial ring in characteristic zero. In the next section, we describe the key features of the package and discuss the methods available for computing decompositions of the invariant ring as in (†). We conclude the article with a demonstration of these features by way of an extended example.

OVERVIEW. The package InvariantRing contains the methods generateGroup, reynoldsoperator, and molienSeries as tools for studying the invariant ring of a finite group. To aid the input of finite groups of matrices, the method generateGroup takes a list of generating matrices $\left\{A_{1}, \ldots, A_{m}\right\}$ and outputs the group they generate, using a brute-force algorithm that computes all possible products of the $A_{i}$. The method reynoldsoperator uses a stored group $G$ to compute the average $\frac{1}{|G|} \sum_{A \in G} A \cdot f$ of a polynomial $f \in S$. Finally, the method molienseries computes the Hilbert series $H\left(S^{G}, t\right)$ of $S^{G}$ using Molien's theorem, expressing the result as a rational expression of the divide class.

The core of the package consists of methods for computing a decomposition as in (\$). The method primaryInvariants implements two different algorithms for computing primary invariants for $S^{G}$ : the 'optimal' algorithm from [K2] and the 'Dade' algorithm, described in [DK, S], for example. The first algorithm is the default for the method primaryInvariants. It begins by cycling through $n$-tuples $\left(d_{1}, \ldots, d_{n}\right) \in \mathbb{N}^{n}$, ordered by increasing values of the product $d_{1} \cdots d_{n}$. For each tuple, it tests whether primary invariants with degrees $d_{1}, \ldots, d_{n}$ can exist, firstly by using the two conditions from Theorem 2 and then by using the Krull dimension test from [K2, Theorem 2(b)]. If these tests are passed, a set of primary invariants with the proposed degrees is constructed iteratively using the same dimension test; otherwise, the next degree vector is considered. The resulting primary invariants have coefficients that are integers or uncomplicated fractions. In addition, the product of their degrees is as small as possible, so the number and degrees of secondary invariants needed in ( $\$)$ are kept small, by Theorem 2 .

The default routine in the method primaryInvariants cannot compute primary invariants when working over ground fields of positive characteristic, even if the characteristic is coprime to $|G|$. In this case, the method primaryInvariants can be used with the option Dade set to true to find a system of primary invariants using the Dade algorithm instead, provided the cardinality of the ground field is sufficiently large. The algorithm works by finding a 'Dade basis' $v_{1}, \ldots, v_{n}$ of $S_{1}$, then computing $f_{i}=\prod\left\{w \mid w \in \operatorname{Orb}_{G}\left(v_{i}\right)\right\}$ for each $i$; the collection $f_{1}, \ldots, f_{n}$ is then a set of primary invariants. The construction of a Dade basis involves only choosing random linear forms and linear algebra, so has the advantage of being simple and quick. However, the resulting primary invariants almost always have ugly coefficients and have degrees being the same as the order of the group. When over a characteristic zero field with a small group, the Dade algorithm is often faster than the default algorithm, but at the cost of calculating more secondary invariants to obtain a decomposition as in (†).

The method secondaryInvariants uses the primary invariants $f_{1}, \ldots, f_{n}$ and their degrees $d_{1}, \ldots, d_{n}$ to compute corresponding secondary invariants, by finding a collection of $r=d_{1} \cdots d_{n} /|G|$ homogeneous invariants whose degrees are the values predicted in Theorem 2(a) and whose images under the projection $S \rightarrow S /\left(f_{1}, \ldots, f_{n}\right)$ are linearly independent over $k$ (for a justification, see [DK, §3.5.1]). 
Thus, finding secondary invariants boils down to a question of doing linear algebra with normal forms of polynomials with respect to the ideal $\left(f_{1}, \ldots, f_{n}\right) \subset S$. The implementation of this procedure in secondaryInvariants only works when the ground field is of characteristic zero.

The package also includes the method invariantRing for computing a decomposition as in ( directly, based only on the data of the polynomial $\operatorname{ring} S$ and the group $G$. It outputs a collection of primary invariants, computed using Kemper's 'optimal' algorithm from [K2], and a corresponding collection of secondary invariants.

As indicated above, the package InvariantRing cannot compute the full invariant ring of a finite group over ground fields of positive characteristic. However, there are algorithms in the literature that could be implemented in the future to address this. In particular, [K1, K2] give algorithms for computing primary and secondary invariants of $S^{G}$ when $\operatorname{gcd}(|G|, \operatorname{char} k)=1$. When $\operatorname{char} k$ divides $|G|$, the ring $S^{G}$ is in general not Cohen-Macaulay [K1, Example 13], so does not admit a decomposition as in ( $\ddagger)$. In this case, the algorithms in [K1,K2] compute homogeneous, algebraically independent invariants $f_{1}, \ldots, f_{n}$, together with homogeneous invariants $g_{1}, \ldots, g_{r}$ generating $S^{G}$ as a (not necessarily free) $k\left[f_{1}, \ldots, f_{n}\right]$-module.

An EXAMPLE. We conclude this article with an example that demonstrates the main methods available in InvariantRing and compares the default and Dade algorithms for calculating primary invariants. The group $G$ considered is the dihedral group of order 8 , generated by matrices

$$
A=\left[\begin{array}{lll}
-1 & 1 & 0 \\
-1 & 0 & 1 \\
-1 & 0 & 0
\end{array}\right], \quad B=\left[\begin{array}{ccc}
0 & -1 & 1 \\
-1 & 0 & 1 \\
0 & 0 & 1
\end{array}\right] .
$$

This group acts on the polynomial ring $S=\mathbb{Q}[x, y, z]$.

To begin, the print width is altered, the package InvariantRing is loaded, and the matrices and polynomial ring input.

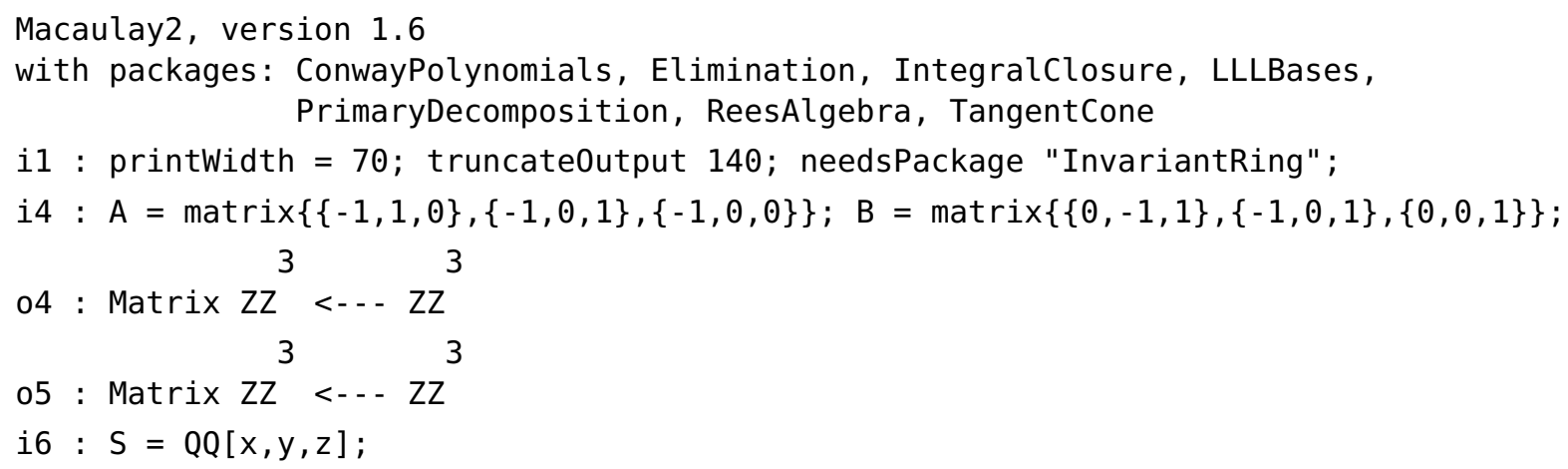

We generate a group $G$ from the list of matrices $\{A, B\}$ using the generateGroup method. The field in the second argument ensures the resulting matrices are defined over $\mathbb{Q}$, the ground field of $S$.

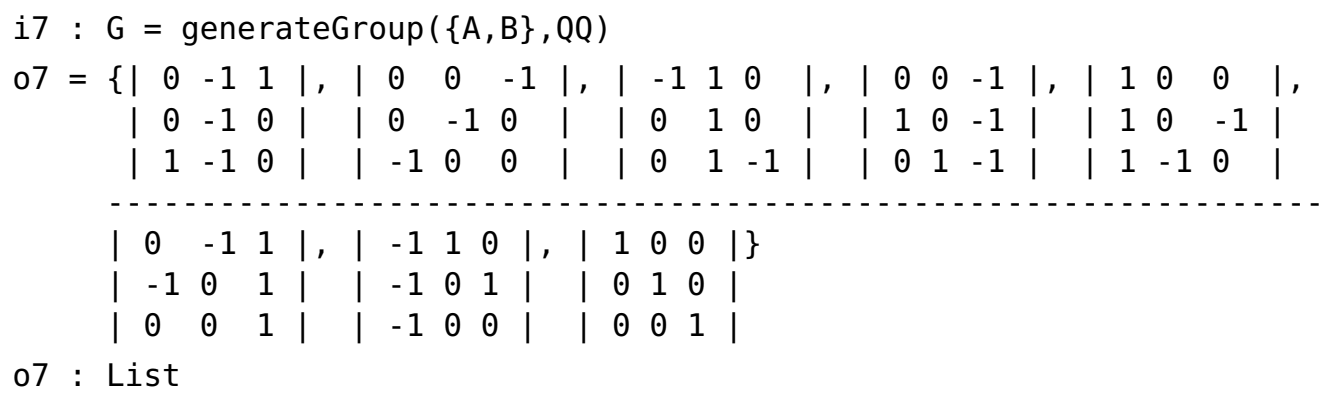


Next, we find a list of primary invariants for $S^{G}$ using primaryInvariants. We do this using both the default algorithm and the Dade algorithm, the latter being called by setting the optional argument Dade to true. We obtain the degrees of the primary invariants computed with the Dade algorithm, but truncate the full output.

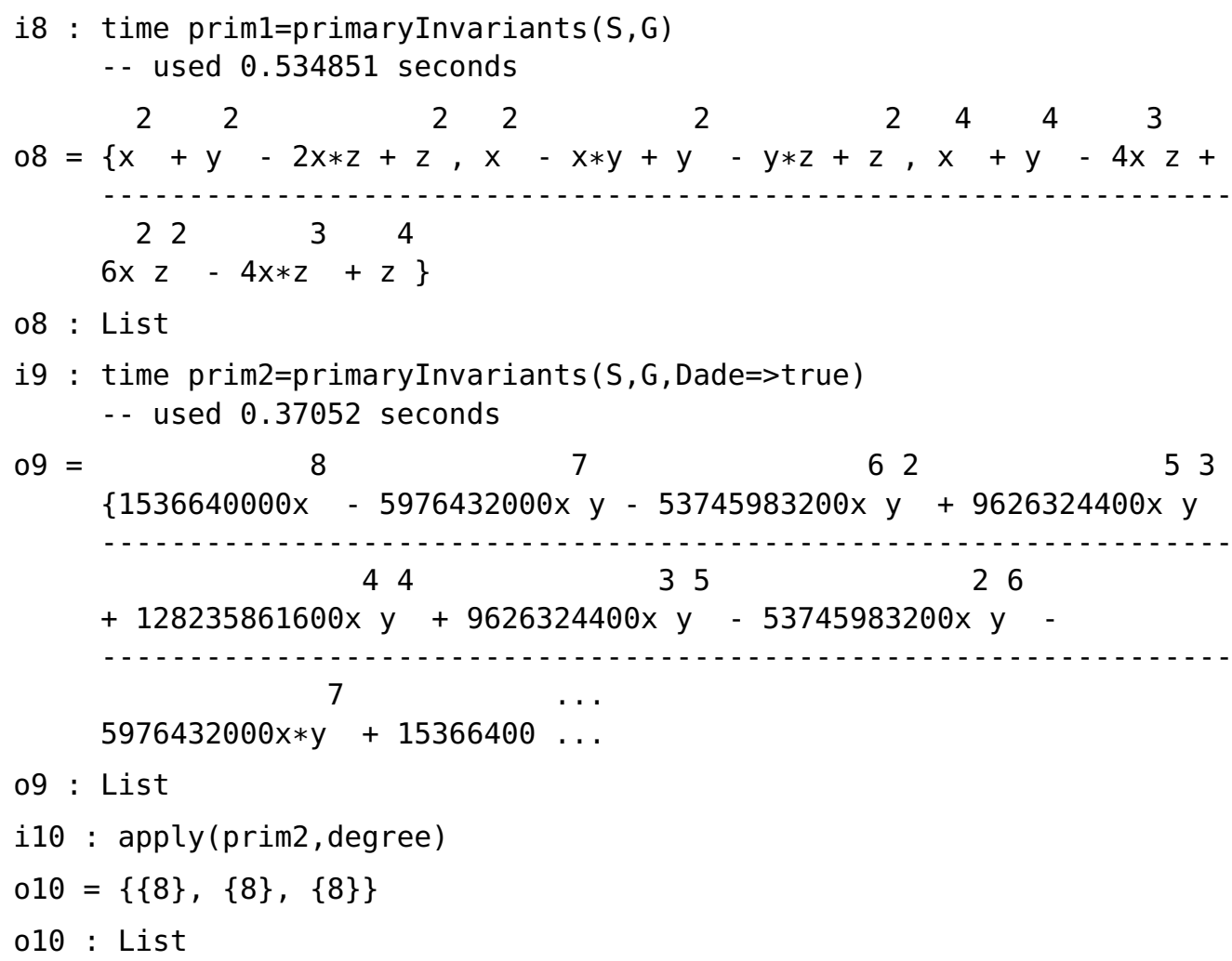

The Dade algorithm was faster than the default algorithm in this example, but the resulting invariants are complicated polynomials of degrees equalling the order of the group, which will influence the time needed to compute secondary invariants. This is done next by applying the method secondaryInvariants to the lists prim1 and prim2. According to Theorem 2 (b) there are $8^{3} / 8=64$ secondary invariants corresponding to the collection prim2, so again we suppress the output.

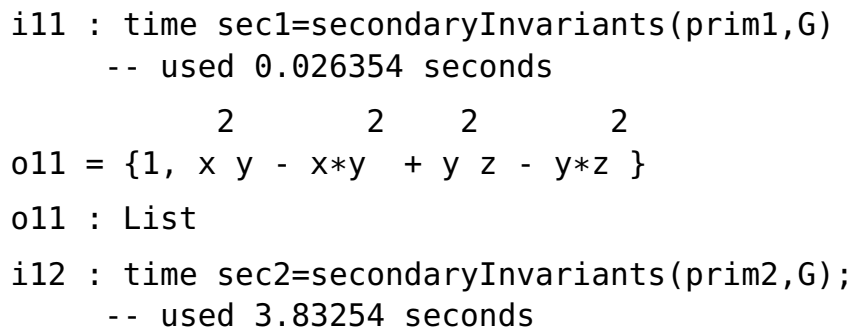

We note how much quicker it was to compute the secondary invariants for prim1 than prim2. In fact, on this occasion the default algorithm for computing primary invariants resulted in a quicker overall computation of the decomposition ( $\$$ ) for the invariant ring of $G$.

To see how many corresponding secondary invariants of each degree there are, we compute the Hilbert series of $S^{G}$ using the method molienseries and Theorem 2(a).

i13 : $\mathrm{mol}=$ molienSeries $\mathrm{G}$ 


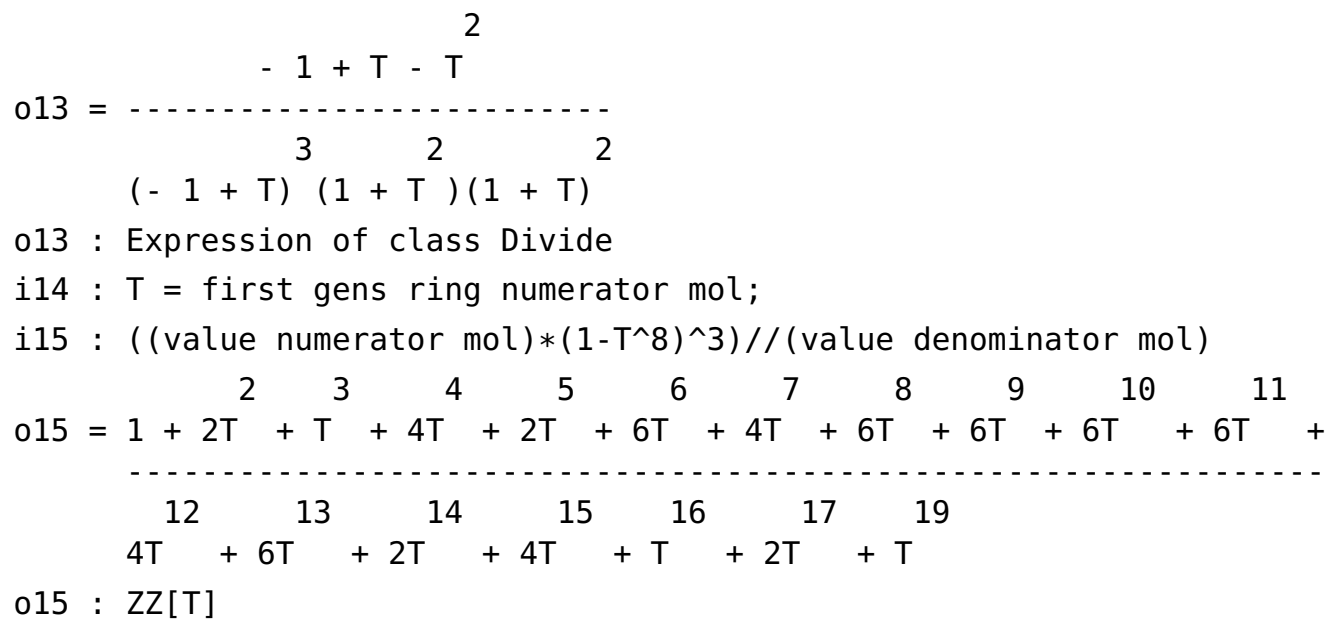

Thus, for example, we see that there are 6 secondary invariants of degree 11 . To print this polynomial during the construction of secondary invariants, we call secondaryInvariants with the optional argument PrintDegreePolynomial set to true:

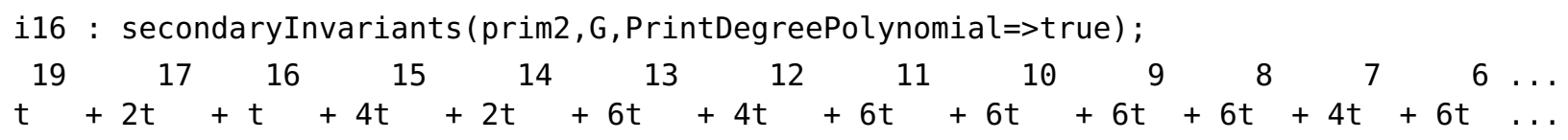

Finally, we mention that the method invariantRing computes primary and secondary invariants in one go, calling upon the method primaryInvariants with the optional argument Dade set to false. Executing the command invariantRing $(S, G)$ in the above example would output \{prim1, sec1\}.

Acknowledgement. The author wishes to express his gratitude to Diane Maclagan for suggesting and supervising the writing of this package. He also thanks an anonymous referee and Amelia Taylor for helpful comments concerning the code and this article.

\section{REFERENCES.}

[DK] H. Derksen and G. Kemper, Computational invariant theory, Invariant Theory and Algebraic Transformation Groups, I, Springer-Verlag, Berlin, 2002.

[K1] G. Kemper, Calculating invariant rings of finite groups over arbitrary fields, J. Symbolic Comput. 21 (1996), no. 3, 351-366.

[K2] __ An algorithm to calculate optimal homogeneous systems of parameters, J. Symbolic Comput. 27 (1999), no. $2,171-184$.

[M2] D.R. Grayson and M.E. Stillman, Macaulay2, a software system for research in algebraic geometry, available at www. math. uiuc. edu/Macaulay2/.

[N] M.D. Neusel, Invariant theory, Student Mathematical Library, vol. 36, American Mathematical Society, Providence, RI, 2007.

[S] B. Sturmfels, Algorithms in invariant theory, 2nd ed., Texts and Monographs in Symbolic Computation, SpringerWienNew York, Vienna, 2008.

RECEIVED : 2012-08-10 REVISED : 2013-03-24 ACCEPTED : 2013-05-16 